\title{
Some Thoughts on Cancer Research
}

\author{
By R. KEITH CANNAN, D.Sc.
}

$\mathrm{T}$ HE OBJECTIVE of cancer research is the control of cancer in man. This statement would appear to be so obvious as to require no elaboration. The inquiring layman, however, who surveys contemporary programs of research sponsored by agencies interested in the control of cancer may well find occasion to pause. He will observe that a large proportion of the work currently in progress is being conducted in laboratories rather than at the bedside, and he will note also that much of it is concerned with problems that seem to bear no apparent relation to human cancer. He may well wonder whether investigators are losing sight of the medical woods in their zest for climbing their own particular scientific trees.

Why is cancer research diffused so widely over the face of medical science, and why is much of it so remotely related to the cancer patient? The question is a pertinent one and merits thoughtful answer.

In this matter, as in most critical appraisals, it is well to begin by laying down some basic propositions. Two will suffice for the argument that follows.

Dr. Cannan is chairman of the Division of Medical Sciences, National Research Council. His discussion of cancer research was prepared at the suggestion of the National Cancer Institute, Public Health Service, and is based on his testimony at the health inquiry hearing of the Committee on Interstate and Foreign Commerce, House of Representatives, October 2, 1953.
The first proposition is that cancer is a disease of individual cells. The weight of evidence forces the conclusion that a tumor is merely the visible sign of the presence in a tissue of colonies of aberrant cells that have broken through the normal restraints on cell growth and are multiplying without regard to the health of the tissue from which they came or the economy of the organism as a whole. They are, however, not merely normal cells "on the loose." They are specifically and irreversibly modified cells. They are disordered rather than disorderly. This conclusion is implicit in the fact that their abnormal characteristics are such an intimate part of the cells that they are passed on to their progeny in the course of cell division. Cancer is a self-multiplying disorder of cells and therein lies its malignancy.

The second proposition is that the living cell is a chemical machine. A cell is recognized microscopically by its characteristic structure, but it is much more than an inert structure. It is a process. A living cell is a "becoming" rather than a "being." It lives by reason of a constant flux of chemical change whereby energy is mobilized for growth and multiplication and for the contribution of the individual cell to the activities of the organism as a whole. The structure of a cell, like that of an inanimate machine, is simply an organization of parts adapted to the purpose of converting energy into specific processes in an orderly and efficient manner. Each type of cell has its own peculiar structure designed to effect its own particular processes. Each type of cell has its own pattern of chemical activity. Change in structure or 
change in physiological function is a reflection of some basic change in this complex of chemical reactions. In the last analysis the understanding and control of cellular activity is a biochemical problem.

\section{A Biochemical Problem}

If these two propositions are accepted, it follows that a malignant cell must differ chemically from a normal cell because it behaves differently. Since it continues to multiply under conditions in which its neighbors have ceased to do so, the cancer cell must be pursuing a pattern of chemical behavior different from that of the community of normal cells. If this is so, then the chief hope for the control of malignant growths that are beyond the reach of the surgeon's knife or the X-ray beam is the control of their chemical reactions.

Ten or fifteen years ago this conclusion would have been an affirmation of faith without proof because so little was known of the intimate chain of chemical events within the cell. Today, it may be asserted with some confidence that there is accumulating evidence that malignant cells do differ chemically from normal cells. This information has not yet provided us with a workable formula for controlling the growth of cancers, but it does justify the hope that chemical means to do so will be found.

The emphasis which has been placed on the biochemical approach to cancer does not detract in any degree from the importance of biological studies that do not involve chemical techniques. The relation of hereditary factors to cancer, the transplantability of cancers, the transmission of tumors by viruslike agents, the growth of cancer cells in tissue culture, the influence of hormones on susceptibility to the growth of tumors, the attempts to develop antibodies to malignant cells - all these and many other types of study are essential contributions. Without them the biochemist would have nothing to investigate. In the final issue, however, cancer is a biochemical problem, and the ultimate objective of those who are pursuing the biological avenues of approach indicated above must be to find chemical descriptions for the phenomena that they observe.

\section{Obligations of Medicine}

Medicine recognizes two major obligations. The first is the diagnosis and cure of disease as and where it is encountered. A more far-reaching obligation, however, is the prevention of disease by the disclosure and control of its causes. Much of the contemporary research in the field of cancer appears to be directed toward the search for the causes of malignant growth rather than toward the refinement of the therapeutic measures that offer the greatest immediate promise. There has been some criticism of this distribution of effort, but the criticism ignores the fact that the two approaches are not competitive but complementary. The worker in the laboratory is constantly developing new ideas, devising new tools, and exploring new concepts, which he refines and passes on to the clinical investigator. The clinical investigator, as constantly, is testing these ideas and applying these tools in the clinic and is passing back new questions, thereby channeling the work in the laboratory. A remarkable aspect of clinical investigation today is the speed with which new knowledge, garnered in the laboratory, receives clinical appraisal. In point of fact the seekers after causes and the seekers for diagnostic tests and therapeutic measures may pose their questions differently, but they find themselves exploring the same ground-the ground of the biochemistry of the cell. It is quite probable that the next major advance in chemotherapy may arise from some observation made in the course of an academic study of the cause of a particular animal tumor. It is also possible that the next new idea about the causes of malignant growth may emerge from clinical studies at the bedside or in the field.

\section{Known Causes}

A few extraneous causes of human cancer are known. They include several types of radiation and a heterogenous group of chemicals comprising the salts of a few metals, certain curious hydrocarbons, some tars, and a few intermediates in the manufacture of dyes. This is a motley assembly. These agents are known to interfere in one way or another with the chemistry of cells, but their chemical effects 
exhibit no common characteristic which would explain their unique property of promoting cancerous growth.

To what extent human cancer is caused by exposure to environmental cancerogens is uncertain. Those enumerated above are relatively minor causes. On the other hand, the alarming increase in lung cancer in males during the past three decades has alerted medicine to the fact that civilized man is increasingly surrounding himself with an unnatural chemical environment whose cancerogenic potentialities have not been explored. Intensive studies of the possible relation of the incidence of particular types of cancer to exposure to chemical contaminants by contact, inhalation, or ingestion are badly needed.

\section{Susceptibility}

Although the known environmental cancerogens are of clinical importance only with respect to limited groups of people who may be subject to undue exposure to one or another of these agents, they have proved to be immensely valuable tools in the laboratory because they make possible controlled studies of the early and late stages in the progress of malignant growth. Such studies have established the fact that the development of a tumor depends not only on the presence of an inciter but also on the susceptibility of the exposed cells. There are wide differences in sensitivity to a particular cancerogen of the various types of cell in a single animal and also of a single type of cell in different species and, even, in different strains of the same species. To add to the confusion the relative susceptibilities of particular cell types to a series of cancerogens are quite variable. Such observations, coupled with the knowledge of the wide differences in the natural histories of the many types of human tumors lead to the inescapable conclusion that cancer is not one but many diseases and can have many causes.

It must be acknowledged that the term "susceptibility" is a cloak for ignorance. In part it represents the inherent resistance of the cell to the action of the inciter and, in part, the extent to which the body as a whole is able to restrain the tendency toward disordered growth that is presumably invoked by the in- citer in susceptible cells. The hormones, and possibly other regulatory mechanisms of the body, are involved in the execution of these restraints. Whatever may be the complex of processes that determine the susceptibility of a cell to a particular inciter, the processes must be chemical in nature because the cell is a chemical machine.

Insofar as human cancer cannot be shown to be environmental in origin, the investigator must look within the body for its causes. In this difficult quest there are few leads. One can only surmise that there must arise in the body of the host some cancerogenic agent which evokes malignant changes in susceptible cells. This inciter may be a product of some chronic disorder of metabolism, some spontaneous distortion of the chemical mechanisms of heredity, or, possibly, an ill-defined agent comparable to the viruslike bodies that are known to be associated with certain animal tumors.

The argument that has been developed above reduces to the simple statement that the goal of the seeker after causes is the disclosure of chemical anomalies antecedent to the overt development of malignant cells. As and when these are uncovered, it will be possible to develop rational approaches to their therapeutic correction or control. In the meantime, attempts to improve present methods of diagnosis and treatment cannot be neglected. The best must not be allowed to be the enemy of the good.

\section{Present Hope}

Cancer can be cured if it is localized and accessible to the surgeon or the radiologist. The urgent challenge of cancer is the detection and control of hidden and diffuse malignancies. Present hope lies in chemotherapy. Chemical agents must be sought which will be carried in the blood stream to the cancer cells and will destroy them or inhibit their growth without disrupting the essential functions of normal tissues.

Faced with millions of available compounds, the investigator will not attempt to proceed on any hit-or-miss basis. He seeks out differences in the chemistry of normal and malignant cells 
in the hope of identifying cellular reactions which are more sensitive to chemical interference in cancer cells than in normal cells. At present the direction taken in most chemotherapeutic studies stems from the argument that, because malignant cells multiply rapidly, the synthetic reactions associated with cell division are the most promising targets. On this basis it is possible to make a rational approach by selecting as potential interfering compounds a restricted group of chemicals that may be expected to interact with the cell constituents whose synthesis seems to control the division of the cell.

Even so the problem is proving to be highly complex. Each type of tumor in animals shows its own range of sensitivities to a series of drugs. Moreover, the same type of cancer in different species varies in its sensitivity to a particular chemotherapeutic agent. Evidently, each type of tumor in man must be investigated as a separate therapeutic problem. It is unlikely that a single miracle drug will be found that will control all of the major cancers that afflict mankind. On the other hand it becomes increasingly probable that medicine will develop a battery of drugs each of which will have therapeutic value under limited conditions.

In the control of human cancer much emphasis has been placed on the value of early diagnosis. Here, again, it is the buried and diffuse tumor that is the urgent problem. The hope is that the chemical disturbances that are presumed to be associated with the precancerous or early cancerous states are reflected in chemical changes in the body fluids and that means of identifying the latter can be devised. As in the case of chemotherapeutic agents it is unlikely that a single test will betray all types of cancer, but there is reason to hope that a series of tests of limited specificity will be evolved.

\section{Conclusions}

In conclusion, one may properly ask the question why the intensive study of the chemistry of cancer has been so long delayed. The answer can only be that the solution of a scientific problem requires tools appropriate for the task. With respect to the biochemistry of cancer, progress waited upon the development of instruments and techniques sensitive and gentle enough to probe the chemical activities of small groups of cells while they are alive and functional. These are now at hand. Specifically, the emergence of radioactive isotopes as molecular tracers has been the master key that is unlocking the doors of the biochemistry of the cell.

Notable advances have been made during the past decade in all avenues of cancer research. However, the most promising aspect of the contemporary scene is not the accumulation of isolated pieces of information but the fact that the many avenues to knowledge are rapidly drawing together and finding common ground in biochemistry. The biochemist and the biophysicist have now refined their techniques to the point that they are ready to join hands with the clinician, the geneticist, the cytologist, the microbiologist, and the endocrinologist. When scientists using different vocabularies reach common ground and learn to employ the same language, a notable intellectual synthesis has been achieved.

It may be well to emphasize that the timing of this larger synthesis was not at the command of investigators within the field of cancer studies. Its emergence depended upon developments in all the sciences ancillary to medicine. The future of cancer research does not lie in the hands of any group of the elect. It is determined by the progress of science as a whole. Herein lies a moral for those who administer the affairs of medical research. To attempt to encourage the study of single diseases as phenomena isolated from the main stream of medical and biological knowledge is the surest way to impede progress. 